\title{
Anomalia de Ebstein em paciente adulto: valvuloplastia modificada para correção de insuficiência tricúspide
}

\author{
Mauro Barbosa ARRUDA FILHO*, Heraldo MAIA JR., Sérgio RAYOL*, Flávia Arruda SANTOS*, Ana \\ Paola Morais ARRUDA*, Claudia Arruda Buarque de GUSMÃO*, Mauro Barbosa ARRUDA*
}

RBCCV 44205-582

Arruda Filho M B, Maia Jr. H, Rayol S, Santos F A, Arruda A P M, Gusmão C A B, Arruda M B - Anomalia de Ebstein em paciente adulto: valvuloplastia modificada para correção de insuficiência tricúspide. Rev Bras Cir Cardiovasc 2002; 17(2): 24-28.

RESUMO: Objetivos: Avaliar a correção cirúrgica em pacientes adultos portadores de anomalia de Ebstein, utilizando uma variação técnica para correção da insuficiência tricúspide.

Casuística e Métodos: Entre janeiro de 1990 e março de 2001, seis pacientes adultos foram submetidos à correção cirúrgica com uma variação da técnica apresentada por Carpentier. A idade variou de 18 a 34 anos. Todos se apresentavam em classe funcional III ou IV (NYHA), com piora da cianose ou arritmias freqüentes. Em quatro pacientes a valva tricúspide era do tipo $B$ e em dois do tipo A (Carpentier), quatro apresentavam comunicação interatrial (CIA) associada. $O$ hematócrito variou de $33 \%$ a $68 \%$. O índice cardíaco variou de 0.47 a 0.88 . A fração de ejeção do ventrículo esquerdo variou de $50 \%$ a $80 \%$.

Resultados: Não se registrou óbitos no período hospitalar. Um paciente foi reoperado por sangramento no pós-operatório imediato. Em dois pacientes ocorreram derrames pericárdicos de repetição. Foi registrado um óbito no 14ํำ mês de pós-operatório por morte súbita. Os pacientes foram seguidos por um período de 10 a 108 meses. Quatro encontram-se em classe funcional I e um encontra-se em classe funcional II (NYHA). O ecocardiograma no pós-operatório imediato e na última avaliação mostra insuficiência tricúspide discreta ou moderada em quatro e importante em um paciente.

Conclusão: Apesar do pequeno número de pacientes nesta série, a modificação técnica cumpriu o seu papel, com a melhora clínica e funcional dos pacientes tratados em até nove anos de seguimento.

DESCRITORES: Anomalia de Ebstein, cirurgia. Cardiopatias congênitas, cirurgia. Valva tricúspide, cirurgia.

INTRODUÇÃO

Trabalho realizado no Serviço de Cirurgia Cardiovascular e Torácica do Real Hospital Português de Beneficência em Pernambuco. Recife, PE, Brasil.

Recebido para publicação em outubro de 2001.

*Do Real Hospital Português de Beneficência em Pernambuco.

Endereço para correspondência: Mauro B. Arruda Filho. Rua Gervasio Fioravante, 87/202. Graças. Recife, PE, Brasil. CEP 52011-030. Tel: (81) 34235496. e-mail: arrudamf@ hotlink.com.br. 
A anomalia de Ebstein é uma má formação de valva tricúspide, rara, ocorrendo em cerca de $1 \%$ de todas as cardiopatias congênitas ${ }^{(1)}$. Na maioria dos casos, os folhetos posterior e septal da valva apresentam-se inseridos anormalmente no interior do ventrículo direito, tornando a cavidade ventricular atrializada ${ }^{(2)}$, provocando freqüentemente aumento da cavidade atrial, insuficiência tricúspide e disfunção ventricular direita.

O quadro clínico é caracterizado por dispnéia, cianose, arritmias, cardiomegalia e insuficiência ventricular direita, que se manifestam em intensidade e períodos variáveis no curso da doença.

O tratamento da anomalia de Ebstein é eminentemente cirúrgico e consiste na correção da disfunção valvar e correção dos defeitos associados quando coexistam. Atualmente, o defeito valvar é corrigido através de plastias e ou substituição valvar com ou sem plicatura longitudinal da porção atrializada do ventrículo direito, cujos resultados não são uniformes (3-16)

O objetivo do presente trabalho é avaliar os resultados obtidos com uma variação técnica de plastia da valva tricúspide, tomando-se por base a técnica utilizada por Carpentier ${ }^{(8)}$. Nesta variação técnica é realizada a desinserção anular da metade do folheto anterior e sua re-inserção com rotação horária, após se proceder a plicatura longitudinal da parede ventricular atrializada, sem a ressecção em cunha desta parede e redução da circunferência do orifício atriventricular direito, fazendo-se uma anuloplastia sem prótese. Este procedimento foi utilizado em 6 pacientes adultos a partir de 1990.

\section{CASUÍSTICA E MÉTODOS}

\section{Pacientes}

Entre janeiro de 1990 e março de 2001, seis pacientes adultos portadores de anomalia de Ebstein foram submetidos à correção cirúrgica no Serviço de
Cirurgia Cardiovascular e Torácica do Real Hospital Português de Beneficência em PE. Quatro pacientes eram do sexo masculino e dois do sexo feminino. A idade variou de 18 a 34 anos (média 23,3 anos). Cinco eram portadores de comunicação interatrial associada, todos tinham insuficiência tricúspide importante e aumento do átrio direito. Três pacientes apresentavam fibrilação atrial preoperatoriamente, dois, ritmo sinusal e um, bloqueio atrioventricular do segundo grau. O diagnóstico foi estabelecido por ecocardiograma e cateterismo cardíaco.

A indicação cirúrgica se baseou na piora da classe funcional para III ou IV (New York Heart Association), aumento da cianose e aparecimento de arritmias freqüentes. O hematócrito variou entre 33\% e $68 \%$ (média 42\%), o índice cardíaco variou de 0.47 e 0.88 , a fração de ejeção de ventrículo esquerdo variou de $50 \%$ a $80 \%(m=60 \% \pm 10 \%)(1 \mathrm{~mm})$ - Tabela 1 .

\section{Técnica Operatória}

Toracotomia por esternotomia longitudinal, instalação da circulação extracorpórea com perfusão na raiz da aorta e canulação de ambas as veias cavas, hipotermia moderada, proteção miocárdica com cardioplegia anterógrada sangüínea a cada vinte minutos, complementada com soro gelado na cavidade pericárdica. Atriotomia direita ampla, no sentido craniocaudal e análise da valva tricúspide. Em quatro pacientes a valva tricúspide era do tipo $B$ e dois pacientes portadores do tipo A, segundo a classificação de CARPENTIER et al. (8).

O procedimento de correção cirúrgica utilizado é uma variação da técnica descrita por CARPENTIER et al. ${ }^{(8)}$, onde é feita a desinserção anular da metade do folheto anterior. Não é feita a excisão de uma faixa muscular da zona atrializada do ventrículo direito. Realiza-se a plicatura longitudinal da porção atrializada do ventrículo e uma anuloplastia com sutura com fio

TABELA 1. CARACTERÍSTICAS DEMOGRÁFICAS, ACHADOS E SINTOMAS EM 6 PACIENTES PORTADORES DE ANOMALIA DE EBSTEIN SUBMETIDOS A PLASTIA TRICÚSPIDE

\begin{tabular}{cccccccc}
\hline $\begin{array}{c}\text { Número } \\
\text { Paciente }\end{array}$ & Idade & Sexo & $\begin{array}{c}\text { Data } \\
\text { Operação } \\
8 / 1 / 90\end{array}$ & $\begin{array}{c}\text { Seguimento } \\
\text { (meses) }\end{array}$ & $\begin{array}{c}\text { Classe } \\
\text { Funcional }\end{array}$ & $\begin{array}{c}\text { Ritmo Pré- } \\
\text { operatório }\end{array}$ & $\begin{array}{c}\text { Defeito } \\
\text { associado }\end{array}$ \\
\hline 2 & 36 & M & 108 & IV & FA & CIA \\
3 & 18 & M & $16 / 4 / 92$ & Óbito $14^{\circ}$ & IV & SIN & CIA \\
4 & 18 & F & $18 / 7 / 93$ & 98 & III & FA & CIA \\
5 & 20 & M & $10 / 9 / 95$ & 72 & IV & BAVII & CIA \\
6 & 24 & F & $24 / 3 / 99$ & 30 & IV & SIN & - \\
\hline
\end{tabular}

M-masculino; F-feminino; FA- fibrilação atrial; SIN- sinusal; BAVII- bloqueio atrioventricular do segundo grau; CIA- comunicação interatrial. 
de Ethibond 2-0, que tem a finalidade de diminuir o orifício atrioventricular e, por fim, procede-se à reinserção da metade do folheto anterior, deslocando-o no sentido horário (Figura 1). Nos casos onde houve associação com comunicação interatrial, presente em cinco pacientes, foi realizada atriosseptoplastia com auxílio de um enxerto de pericárdio bovino. Atriorrafia direita foi realizada antes do despinçamento da aorta. Normalizados os batimentos cardíacos, a temperatura corporal a $37^{\circ} \mathrm{e}$ a volemia, a circulação extracorpórea (CEC) é interrompida. As cânulas são retiradas e após revisão da hemostasia, realiza-se o fechamento do tórax por planos (Tabela 2).

\section{RESULTADOS}

Não foram registrados óbitos no período hospitalar nesta série de seis pacientes submetidos a plastia da valva tricúspide com esta modificação técnica. Um paciente foi reoperado no pós-operatório imediato por sangramento e em dois pacientes ocorreu derrame pericárdico de repetição que necessitou a realização de janela pericárdica no período hospitalar. Foi registrado um óbito no $14^{\circ}$ mês de pós-operatório por morte súbita.

\section{ESQUEMATIZAÇÃO DAS MODIFICAÇÕES TÉCNICAS DA PLASTIA VALVAR TRICÚSPIDE}

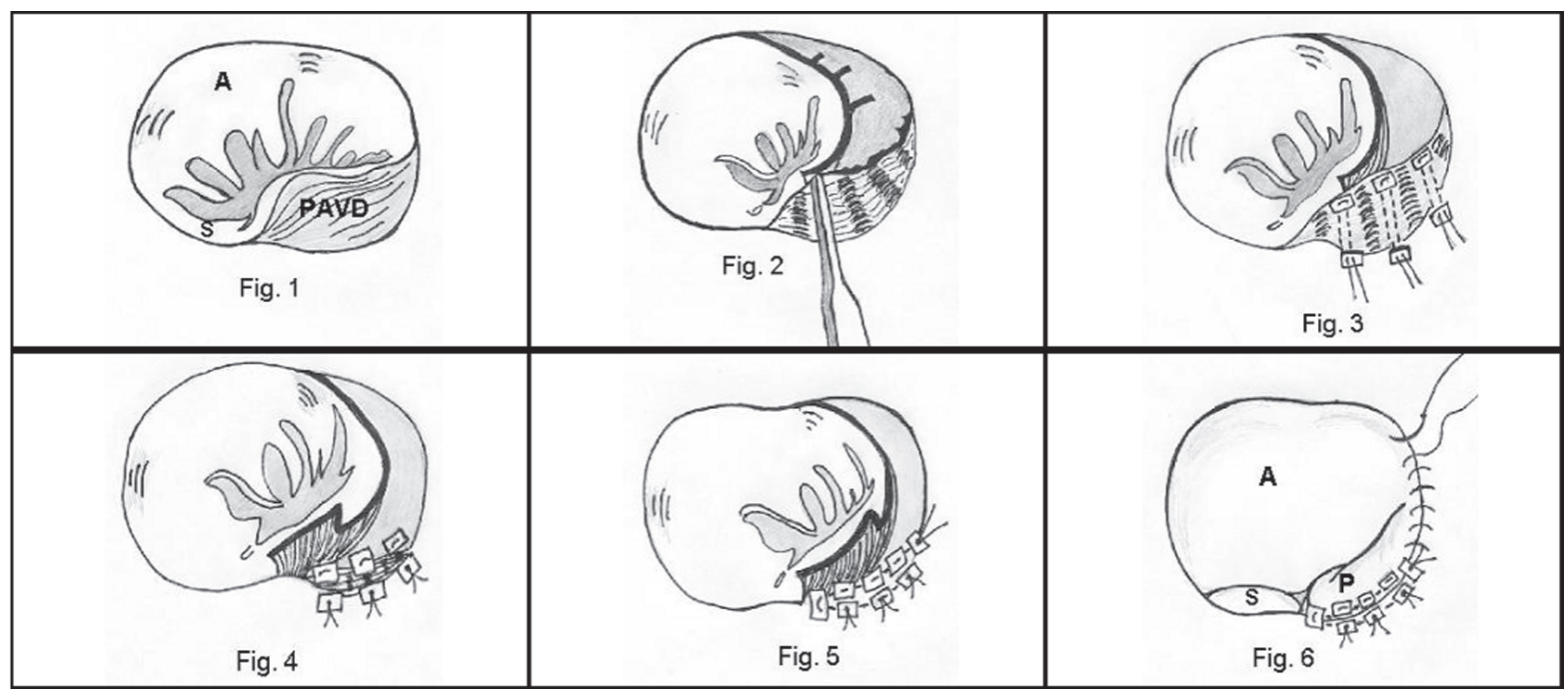

Fig. 1 - A = folheto anterior; $S$ = folheto septal; PAVD = porção atrializada do VD. Fig. 2 - Desinserção do folheto anterior. Fig. 3 - Plicatura longitudinal da PAVD. Fig. 4 - Plicatura longitudinal, diminuição do anel valvar. Fig. 5 - Anuloplastia do folheto anterior. Fig. 6 - Reinserção do folheto anterior - aspecto final.

TABELA 2. ACHADOS INTRAOPERATÓRIOS EM 6 PACIENTES PORTADORES DE ANOMALIA DE EBSTEIN SUBMETIDOS A PLASTIA DA VALVA TRICÚSPIDE

\begin{tabular}{|c|c|c|c|}
\hline $\begin{array}{l}\text { Número } \\
\text { Paciente }\end{array}$ & $\begin{array}{l}\text { Tipo valvar pela } \\
\text { classificação de } \\
\text { Carpentier }\end{array}$ & Reconstituição & $\begin{array}{c}\text { Correção do } \\
\text { defeito associado }\end{array}$ \\
\hline 1 & A & $\begin{array}{l}\text { Plicatura longitudinal+ } \\
\text { anuloplastia sem anel }\end{array}$ & $\mathrm{ClA}$ + enxerto $\mathrm{PB}$ \\
\hline 2 & B & $\begin{array}{l}\text { Plicatura longitudinal+ } \\
\text { anuloplastia sem anel }\end{array}$ & $\mathrm{ClA}+$ enxerto $\mathrm{PB}$ \\
\hline 3 & A & $\begin{array}{l}\text { Plicatura longitudinal+ } \\
\text { anuloplastia sem anel }\end{array}$ & $\mathrm{ClA}$ + enxerto $\mathrm{PB}$ \\
\hline 4 & B & $\begin{array}{l}\text { Plicatura longitudinal+ } \\
\text { anuloplastia sem anel }\end{array}$ & $\mathrm{CIA}+$ enxerto $\mathrm{PB}$ \\
\hline 5 & B & $\begin{array}{l}\text { Plicatura longitudinal+ } \\
\text { anuloplastia sem anel }\end{array}$ & - \\
\hline 6 & B & $\begin{array}{l}\text { Plicatura longitudinal+ } \\
\text { anuloplastia sem anel }\end{array}$ & $\mathrm{ClA}+$ enxerto $\mathrm{PB}$ \\
\hline
\end{tabular}

CIA -comunicação interatrial; PB - pericárdio bovino. 
Cinco pacientes foram seguidos clinicamente por um período de 10 a 108 meses. A curva actuarial de sobrevida em um ano foi de $100 \%$ e $83.3 \% \pm 5,6 \%$ em 9 anos. Quatro pacientes encontram-se em classe funcional III ou IV da New York Heart Association e 1 encontrase em classe funcional II (NYHA) - (Tabela 1).

O ecocardiograma transtorácico no momento da alta, após um ano e na última avaliação, mostrou que quatro pacientes apresentam insuficiência tricúspide discreta ou moderada e um apresenta insuficiência importante, porém mantém-se em classe funcional II (NYHA).

O índice cardiotorácico regrediu em quatro $(80 \%)$ dos cinco pacientes em seguimento. Três mantêm-se em ritmo sinusal, um em ritmo juncional e um em fibrilação atrial. Nenhum episódio de arritmia supraventricular foi diagnosticado.

Não houve alteração significativa da fração de ejeção do ventrículo esquerdo nos períodos pré-operatório e no seguimento após a correção $(60 \% \pm 10 \%$ X 59\% \pm 8\%).

\section{COMENTÁRIOS}

Uma modificação técnica de plastia da valva tricúspide na correção da anomalia de Ebstein, tomando por base a técnica descrita por CARPENTIER et al. (8), foi realizada em seis pacientes adultos. Em princípio, as técnicas de plastia descritas na literatura estão sempre na dependência do tamanho e da mobilidade do folheto anterior ${ }^{(10-15)}$.

A multiplicidade de apresentação do defeito valvar deu origem a uma classificação anatômica da valva tricúspide malformada, proposta por CARPENTIER et al. ${ }^{(8)} \mathrm{em}$ quatro tipos: A, B, C e D. O tipo A refere-se a casos onde há médio a moderado deslocamento da origem dos folhetos septal e posterior, havendo preservação da cavidade trabeculada do ventrículo direito com pequena área atrializada. No tipo B a relação é invertida. Há uma grande cavidade atrializada e um pequeno ventrículo contrátil. Contudo, em ambos os tipos, o folheto anterior é grande e com boa mobilidade. No tipo C, o folheto anterior tem uma mobilidade limitada, por aderência à parede anterior do ventrículo direito, por bandas fibrosas e ou cordas anômalas. O tipo D é chamado de saco tricúspide ${ }^{(16)}$, é descrito em casos onde, praticamente, o interior do ventrículo direito é revestido por restos fibrosos da valva tricúspide.

De acordo com esta classificação, dois dos pacientes operados eram do tipo A e quatro eram do tipo B. A modificação técnica aplicada acrescenta a não excisão cirúrgica de parte da zona atrializada do ventrículo direito, a realização de uma anuloplastia por sutura de $1 / 4$ do anel posterior da valva tricúspide, com a finalidade de diminuir o orifício atrioventricular. Além disso, a plicatura longitudinal da parede atrializada do ventrículo direito, descrita anteriormente por DANIELSON et al. (12), e, finalmente, o reimplante da metade do folheto anterior previamente desinserido, originalmente descrito por CARPENTIER et al. ${ }^{(8)}$.

Os resultados obtidos nesta pequena série de pacientes, após um seguimento de 9 a 108 meses, são semelhantes aos encontrados na literatura, confirmando que a modificação técnica cumpriu sua finalidade de corrigir a insuficiência tricúspide sem trazer transtornos clínicos aos pacientes, oferecendo bons resultados e pequenos riscos. Evidentemente, faz-se necessário a utilização da técnica em séries maiores, com seguimento clínico prolongado.

RBCCV 44205-582

Mauro Barbosa ARRUDA FILHO*, Heraldo MAIA JR., Sérgio RAYOL* Flávia Arruda SANTOS*, Ana Paola Morais ARRUDA*, Claudia Arruda Buarque de GUSMÃO*, Mauro Barbosa ARRUDA - Ebstein's anomaly in adult patients: modified repair technique for tricuspid insufficiency. Rev Bras Cir Cardiovasc 2002; 17(2): 23-27.

ABSTRACT: Purpose: To value the surgical treatment of adult patients with Ebstein's anomaly, using a Carpentier's modified repair technique.

Material and Methods: Between January 1990 and March 2001 six adult patients with Ebstein's anomaly were treated using a modification of Carpentier's repair technique, four were males and two were females, ages ranging from 18 to 34 years (Mean 23.3 years), all were in Functional Class III or IV (NYHA), with increased cyanoses and frequent rhythm disturbance. Four patients had an associated atrioventricular septal defect; in four patients the tricuspid valve was Carpentier's type B and two type A and all were able to benefit from this conservative technique.

Results: There were not hospital deaths and one patient died $14^{\circ}$ months after surgery in this series. Actuarial survival was $83.3 \%$ in 9 years. Four patients are in Functional Class I and one in Functional Class II (NYHA). Echocardiography and Doppler studies demonstrated a normal shape of right ventricle and good tricuspid valve function in four patients and one has moderate tricuspid regurgitation but still in Functional Class II (NYHA).

Conclusion: Although the small number of patients in this series, the modification of Carpentier's repair technique in adult patients was efficient, reproducible and improved the preoperative status with regard to functional class, tricuspid regurgitation cyanoses and rhythm disturbance.

DESCRIPTORS: Ebstein's Anomaly, surgery. Heart defects, congenital, surgery. Tricuspid valve, surgery. 


\section{REFERÊNCIAS BIBLIOGRÁFICAS}

1 Rangel A, Perez J, Badui, E et al. - Distribution of frequency, age, gender and pulmonary arterial pressure. Arch Inst Cardiol Méx 1997; 67: 307-22.

2 Ebstein WU - Einen sehr seltenen fall von insufficienz der valvula tricuspidalis, beding eine ange borene hochogradie missbilding deselben. Arch Anat Physiol Wiss Med 1866; 238-55.

3 Augustin N, Schmidt-Habelmann P, Wottke M, Meisner $\mathrm{H}$, Sebening $\mathrm{F}$ - Results after surgical repair of Ebstein's anomaly. Ann Thorac Surg 1997; 63:1650-6.

4 Barbero-Marcial M, Verginelli G, Awad M, Ferreira S, Ebaid M, Zerbini EJ - Surgical treatment of Ebstein's anomaly: early and late results in twenty patients subjected to valve replacement. $J$ Thorac Cardiovasc Surg 1979; 78: 416-22.

5 Bockeria L A, Kagramanov I I, Kokshenev I V et al. - Immediate results of the Bioglis valve use in the surgical treatment of Ebstein's anomaly. $J$ Heart Valve Dis 2000; 9: 674-7.

6 Caralps JM, Aris A, Bonnin JO, Solanes H, Torner M - Ebstein's anomaly: surgical treatment with tricuspid replacement without right ventricular plication. Ann Thorac Surg 1981; 31: 277-80.

7 Chauvaud S - Ebstein's malformation: surgical treatment and results. Thorac Cardiovasc Surg 2000; 48: 220-3.

8 Carpentier A, Chauvaud S, Mace L et al. - A new reconstructive operation for Ebstein's anomaly of the tricuspid valve. J Thorac Cardiovasc Surg 1988; 96: 92-101.
9 Tanaka M, Ohata T, Fukuda S, Kigawa I, Yamashita $Y$, Wanibuchi $Y$ - Tricuspid valve supraanular implantation in adult patients with Ebstein's anomaly. Ann Thorac Surg 2001; 71: 582-6.

10 Hardy L, May A, Webester A et al. - Ebstein's anomaly: a functional concept and successful definitive repair. J Thorac Cardiovasc Surg 1964; 48: 927-40.

11 Hunter W \& Lillehei W - Ebstein's malformation of the tricuspid valve: study of a case, together with suggestion of a new form of surgical therapy. Dis Chest 1958; 33: 297-304.

12 Danielson K, Maloney JD, Devloo RA - Surgical repair of Ebstein's anomaly. Mayo Clin Proc 1979; 54: 185-92.

13 Quaegebeur JM, Sreeram N, Fraser AG et al. - Surgery for Ebstein's anomaly: the clinical and echocardiographic evaluation of a new technique. $J$ Am Coll Cardiol 1991; 17: 722-8.

14 Shore DF, Wong P, Paneth M, Buckley MJ Results of mitral valvuloplasty with a suture plication technique. J Thorac Cardiovasc Surg 1980; 79: 349-57.

15 Danielson GK, Driscoll DJ, Mair DD, Warnes CA, Oliver WC Jr. - Operative treatment of Ebstein's anomaly. J Thorac Cardiovasc Surg 1992; 104: 1195202.

16 Hertzer R, Nagdyman N, Ewert $P$ et al. - A modified repair technique for tricuspid incompetence in Ebstein's anomaly. J Thorac Cardiovasc Surg 1998; 115: 857-68. 